\title{
The Application of Medicinal Plants in Traditional and Modern Medicine: A Review of Thymus vulgaris
}

\author{
Saleh Hosseinzadeh ${ }^{1}$, Azizollah Jafarikukhdan ${ }^{2}$, Ahmadreza Hosseini ${ }^{3}$, Raham Armand ${ }^{4}$ \\ ${ }^{1}$ Medicinal Plants Research Center, Yasuj University of Medical Sciences, Yasuj, Iran \\ ${ }^{2}$ Department of Biology, Faculty of Sciences, University of Yasuj, Yasuj, Iran \\ ${ }^{3}$ Department of Marine Biotechnology, Xiamen University, Xiamen, China \\ ${ }^{4}$ Department of Biology, Faculty of Sciences, University of Behbahan, Behbahan, Iran \\ Email: " $\underline{\text { hosseinzadehsaleh@gmail.com }}$
}

Received 15 June 2015; accepted 12 September 2015; published 15 September 2015

Copyright @ 2015 by authors and Scientific Research Publishing Inc.

This work is licensed under the Creative Commons Attribution International License (CC BY). http://creativecommons.org/licenses/by/4.0/

(c) (i) Open Access

\section{Abstract}

Medicinal plants have played an essential role in the development of human culture. Medicinal plants are resources of traditional medicines and many of the modern medicines are produced indirectly from plants. This study illustrates the importance of traditional and modern medicines in the treatment and management of human diseases and ailments. It has been confirmed by WHO that herbal medicines serve the health needs of about 80 percent of the world's population; especially for millions of people in the vast rural areas of developing countries. Meanwhile, consumers in developed countries are becoming disillusioned with modern health care and are seeking alternatives. Thymus vulgaris is a species of flowering plant in the mint family Lamiacea. Thymus is a widely used medicinal plant in food and pharmaceutical industries. Among different species of Thymus, Thymus vulgaris is used more than other species in therapeutic dosage forms. In Traditional medicine $T$. vulgaris is cultivated in many countries by most people especially in rural areas depending on herbal medicines to treat many diseases including inflammation-related ailments such as rheumatism, muscle swelling, insect bites, pains, etc. Also the modern medicine in essential oil of thyme has demonstrated that the compounds have shown anti-inflammatory, antioxidant, antibacterial and antifungal properties. In this review the objective is to consider the past and present value of medicinal plants such as Thymus vulgar is used in traditional and modern medical practices as bioactive natural compounds.

\section{Keywords}

Medicinal Plants, Traditional and Modern Medicine, Thymus vulgaris, Bioactive Compounds

\footnotetext{
${ }^{*}$ Corresponding author.
}

How to cite this paper: Hosseinzadeh, S., Jafarikukhdan, A., Hosseini, A. and Armand, R. (2015) The Application of Medicinal Plants in Traditional and Modern Medicine: A Review of Thymus vulgaris. International Journal of Clinical Medicine, 6, 635-642. http://dx.doi.org/10.4236/ijcm.2015.69084 


\section{Introduction}

Traditional medicine has remained as the most affordable and easily accessible source of treatment in the primary health care system of resource poor communities. The local people have a long history of traditional plant usage for medicinal purposes. The medicinal use of plants is very old. The writings indicate that therapeutic use of plants is as old as 4000 - 5000 B.C. and Chinese used first the natural herbal preparations as medicines. In India, however, earliest references of use of plants as medicine appear in Rig-Veda, which is said to be written between 1600 - 3500 B.C. Later the properties and therapeutic uses of medicinal plants were studied in detail and recorded empirically by the ancient physicians (an indigenous system of medicine) which are a basic foundation of ancient medical science in India [1]. Medicinal plant is an important element of indigenous medical systems in all over the world. The ethno botany provides a rich resource for natural drug research and development [2]. "Traditional" use of herbal medicines implies substantial historical use, and this is certainly true for many products that are available as "traditional herbal medicines". In many developing countries, a large proportion of the population relies on traditional practitioners and their armamentarium of medicinal plants in order to meet health care needs. Although modern medicine may exist side-by-side with such traditional practice, herbal medicines have often maintained their popularity for historical and cultural reasons [3]. Natural products have played an important role throughout the world in treating and preventing human diseases. Natural product medicines have come from various source materials including terrestrial plants, terrestrial microorganisms, marine organisms, and terrestrial vertebrates and invertebrates [4] and its importance in modern medicine has been discussed in different reviews and reports [5]. The value of natural products in this regard can be accessed from: 1) the rate of introduction of new chemical entities of wide structural diversity, including serving as templates for semi synthetic and total synthetic modification, 2) the number of diseases treated or prevented by these substances, and 3) their frequency of use in the treatment of disease. In recent years, the use of traditional medicine information on plant research has again received considerable interest [6]. In recent times, there have been increased waves of interest in the field of research in natural products chemistry. This level of interest can be attributed to several factors, including unmet therapeutic needs, the remarkable diversity of both chemical structure and biological activities of naturally occurring secondary metabolites, the utility of novel bioactive natural compounds as biochemical probes, the development of novel and sensitive techniques to detect biologically active natural products, improved techniques to isolate, purify, and structurally characterize these active constituents, and advances in solving the demand for supply of complex natural products [7]. The World Health Organization (WHO) has also recognized the importance of traditional medicine and has created strategies, guidelines and standards for botanical medicines. Proven agro-industrial technologies need to be applied to the cultivation and processing of medicinal plants and the manufacture of herbal medicines [8]. Medicinal plants are resources of new drugs and many of the modern medicines are produced indirectly from plants. It is estimated that there are more than 250,000 flower plant species. Studying medicinal plants helps to understand plant toxicity and protect human and animals from natural poisons. In this review the objective is to consider the past and present value of medicinal plants such as Thymus vulgaris used in traditional and modern medical practices as bioactive natural compounds.

\section{Plant Geographical Distribution and Its Description}

Thymus vulgaris is a species of flowering plant in the mint family Lamiaceae, native to southern Europe from the western Mediterranean to southern Italy. It is a bushy, woody-based evergreen sub shrub with small, highly aromatic, grey-green leaves and clusters of purple or pink flowers in early summer [9]. Thyme grows well during a temperate to heat, dry, sunny climate, and wherever the plants don't seem to be shaded. Thyme species do best in coarse, rough soils that may be unsuitable for several alternative plants [10].

\section{Background and History of Traditional Herbal Medicine}

The use of plants as medicine goes back to early man. Fossil records date human use of plants as medicines at least to the middle Paleolithic age. Evidences of this early association have been found in the grave of a Neanderthal man buried 60,000 years ago. Pollen analysis indicated that the numerous plants buried with the corpse were all of medicinal value. The earliest known medical document is a 4000-year-old. Sumerian clay tablet that recorded plant remedies for various illnesses. By the time of the ancient Egyptian civilization, a great wealth of information already existed on medicinal plants. Among the many remedies prescribed were man drake for pain 
relief, and garlic for the treatment of heart and circulatory disorders. This information, along with hundreds of other remedies, was preserved in the papyrus about 3500 years ago. Ancient China is also a source of information about the early medicinal uses of plants [11]. Although animal and mineral materials have been used, the primary source of remedies is botanical. Of the more than 12,000 items used by traditional healers, about 500 are uncommon use. Botanical products are used only after some kind of processing, which may include, for example, stir-frying or soaking in vinegar or wine. In clinical practice, traditional diagnosis may be followed by the prescription of a complex and often individualized remedy. Traditional Chinese medicine is still in common use in China. More than half the population regularly uses traditional remedies, with the highest prevalence of use in rural areas. About 5000 traditional remedies are available in China; they account for approximately one fifth of the entire Chinese pharmaceutical market [12]. Many herbal remedies found their way from China into the Japanese systems of traditional healing. Herbs native to Japan were classified in the first pharmacopoeia of Japanese traditional medicine in the ninth century [13]. In India, herbal medicine dates back several thousand years to the Rig-Veda, the collection of Hindu sacred verses. One useful plant from this body of knowledge is snakeroot, used for centuries for its sedative effects. The number of higher plant species on this planet is estimated at 250,000, with a lower level at 215,000 [14] and an upper level as high as 500,000. Of these, only about $6 \%$ have been screened for biologic activity, and a reported $15 \%$ have been evaluated phytochemically. With high through put screening methods becoming more advanced and available, these numbers will change, but the primary discriminator in evaluating one plant species versus another is the matter of approach to finding leads. There are some broad starting points to selecting and obtaining plant material of potential therapeutic interest. However, the goals of such an endeavor are straight forward. Plants have an advantage in this area based on their long-term use by humans (often hundreds or thousands of years) [15].

\section{Herbal Medicines in Developed Countries}

Plants and their metabolites constituents have a long history of use in modern "western" medicine and in certain systems of traditional medicine, and are the sources of important drugs such as atropine, codeine, dioxin, morphine, quinine. Use of herbal medicines in developed countries has expanded sharply in the latter half of the twentieth century. In recent years, the use of traditional medicine information on plant research has again received considerable interest. While the western use of such information has also come under increasing scrutiny and the national and indigenous rights on these resources has become acknowledged by most academic and industrial researchers. Meanwhile, the need for basic scientific investigations on medicinal plants using indigenous medical systems becomes imminent [16]. The desire to capture the wisdom of traditional healing systems has led to a resurgence of interest in herbal medicines [17], particularly in Europe and North America, where herbal products have been incorporated into so-called alternative, "complementary", "holistic" or "integrative" medical systems. Monographs on selected herbs are available from a number of sources, including the European Scientific Cooperative on Phytotherapy [18], German Commission E [19] and the World Health Organization [20]. The WHO monographs, for example, describe the herb itself by a number of criteria (including synonyms and vernacular names) and the herb part commonly used, its geographical distribution, tests used to identify and characterize the herb (including macroscopic and microscopic examination and purity testing), the active principles (when known), dosage forms and dosing, medicinal uses, pharmacology, contra-indications and adverse reactions. During the latter part of the twentieth century, increasing interest in self-care resulted in an enormous growth in popularity of traditional healing modalities, including the use of herbal remedies; this has been particularly true in the USA [21]. In the European market there are a lot of products derived from natural plants, which are recognized to possess different biological properties, such as antioxidant, antiseptic, diuretic, stimulating the central nervous system, sedative, expectorant, digestive, etc. Some of these plants have been used in traditional medicine since ancient times and are available on market as infusions, tablets and/or extracts. Consumers have reported positive attitudes towards these products, in large part because they believe them to be of "natural" rather than "synthetic" origin, they believe that such products are more likely to be safe than are drugs, they are considered part of a healthy lifestyle, and they can help to avoid un necessary contact with conventional "western" medicine [22].

\section{Modern and Traditional Prescription of Herbal Drugs (Thymus vulgaris)}

The pharmacological treatment of disease began long ago with the use of herbs. Although herbalist waned in the 
eighteenth and nineteenth centuries, many of the remedies employed by the herbalists provided effective treatment. Some of these became useful prescriptions as physicians began experimenting with therapeutic agents. William Withering was the first in the medical field to scientific ally investigate a folk remedy. His studies (1775-1785) of foxglove as a treatment for dropsy (congestive heart failure) set the standard for pharmaceutical chemistry. In the nineteenth century, scientists began purify the active extracts from medicinal plants. One breakthrough in pharmaceutical chemistry came when Friedrich isolated morphine from the opium poppy (Papaver somniferum) in 1806. Continuing this progress, Justus von Liebig, a German scientist became a leader in pioneering the field of pharmacology. With increased knowledge of active chemical ingredients, the first purely synthetic drugs based on natural products were formulated in the middle of the nineteenth century [22]. In plants, these compounds are mostly secondary metabolites under title bioactive compounds such as alkaloids, steroids, tannins, and phenol compounds, flavoneids which are capable of producing definite physiological action on body [23]. The medicinal plants are rich in secondary metabolites and essential oils of therapeutic importance. The important advantages claimed for therapeutic uses of medicinal plants in different ailments are their safety besides being economical, effective and their easy availability. Because of these advantages the medicinal plants have been widely used by the traditional medical practitioners in their day-to-day practice. Among all plant secondary metabolites which act as antioxidants phenol compounds form a large and varied group. Phenol compounds contribute significantly to the antioxidant potential of several plant species [24]. Among different Thymus species, $T$. vulgaris is cultivated in many countries by most people especially in rural areas depend on herbal medicines to treat many diseases including inflammation-related ailments such as rheumatism, muscle swelling, insect bites, pains and so on [25]. T. vulgaris is used more in pharmaceutical dosage forms because it contains more essential oil than other species with high amount of thyme which exhibits considerable anti-tissue and expectorant effects [26]. Considering the fact that Thymus vulgaris is more expensive, some herbal industries tend to use other species of Thymus with different components. So it is crucial to develop a suitable and reliable identification method to confirm the quality of extracts and herbal drugs. Separation and detection of different constituents in plants have been always complicated. Thymus has approved expectorant, anti-tissue and antispasmodic activities. Its antiseptic property is estimated to be 25 times more effective than phenol, with less toxicity [27]. Different species of Thymus are different in content and type of components. Generally they contain thyme, carvacrol, flavonoids and phenol compounds such as rosmarinic acid which may have antiedemic and macrophage-inhibiting effects [28]. Thymus vulgaris shows a polymorphic variation in monoterpene production, the presence of intra-specific chemo type variation being common in the genus Thymus. Each of the six chemo types, geraniol (G), $\alpha$-terpineol (A), thuyanol-4 (U), linalool (L), carvacrol (C), and thymol (T), is named after its dominant monoterpene [27]. Many pharmacological in vitro experiments carried out during the last decade revealed well defined pharmacological activities of both, the thyme essential oil and the plant extracts. The non-medicinal use of thyme is worthy of attention, because thyme is used in the food and aroma industries; it is widely used as culinary ingredient and it serves as a preservative for foods especially because of its antioxidant effect. Thyme essential oil constitutes raw material in perfumery and cosmetics due to a special and characteristic aroma. Thymus vulgaris oil is a combination of monoterpenes and those will acts as anti-oxidative, antimicrobial, medicinal drug, anti-tissue, antispasmodic, and antibacterial activities [29].

\section{Chemical Composition of the Essential oil of Thymus vulgaris}

The essential oil from $T$. vulgaris showed a high content of oxygenated monoterpenes (56.53\%) and low contents of monoterpene hydrocarbons (28.69\%), sesquiterpene hydrocarbons (5.04\%) and oxygenated sesquiterpenes (1.84\%). The predominant compound among the essential oil components was thymol (51.34\%) while the amount of all other components of the oil was less than $19 \%$ [30].

\section{Medicinal Applications}

Thyme has been thought of to be antiseptic, antimicrobial, medication, astringent, medicinal drug, carminative, disinfectant, medicinal drug and tonic. Thyme is incredibly useful in cases of assorted intestinal infections and infestations, like hook worms, gram-positive and gram-negative bacterium. Its active constituent, thymol, is active against coccid bacteria. Thyme may also improve liver functioning, and act as an appetite stimulant. It will be used in treatment of cartilaginous tube, bronchial and urinary infections [31]. Thyme is helpful in treatment 
of laryngitis and inflammation. The main component of the volatile oil of thyme, thymol, is active against coccid bacteria. It is used for skin issues like oily skin, sciatica, acne, dermatitis, skin condition and bug bites. In aroma therapy, the distinct types, thymol, "red thyme oil", linalool kind for its terribly light soft action for antiviral properties are used. A corrected product, "white thyme oil" is also used, and it's milder on the skin. Applied to the skin, thyme relieves bites and stings, and relieves neuralgia and rheumatic aches and pains [32].

\subsection{Anti-Oxidant Properties}

An antioxidant is a molecule that inhibits the oxidation of different molecules. Oxidation is a chemical process that transfers electrons or hydrogen from a substance to an oxidizing agent. Oxidation reactions will produce free radicals. In turn, these radicals will begin chain reactions. Once the chain reaction happens in a cell, it will cause damage or death to the cell. Antioxidants stop these chain reactions by removing free radical intermediates, and inhibit different oxidation reactions. The leafy parts of Thymus vulgaris and its essential oil have been used in foods for the flavor, aroma and preservation and also in folk medicines. The components of Thymus vulgaris essential oil and the protective effects of this oil against aflatoxin-induce oxidative stress had been studied in some researches. The results indicated that the oil contains carvarcrol (45 mg/g), Thymol (24.7 mg/g), $\beta$-Phellandrene (9.7 mg/g), essential oil (4.1 mg/g), Humuline (3.1 mg/g), $\alpha$-Phellandrene (2.3 mg/g) and Myrcene (2.1 $\mathrm{mg} / \mathrm{g}$ ) [33]. However, $\alpha$ and $\beta$-pinene, Myrcene, $\alpha$-thyjone, Tricyclene, 1,8 -cineole, and $\beta$-sabinene were found in very lower concentrations. Treatment with aflatoxins alone disturbs lipid profile in blood serum, decreases total antioxidant capability, increase uric acid and nitric oxide in blood serum and lipid per-oxidation in liver and excretory organ attended with a sever histological changes within the liver tissues. The oil alone at the 2 tested doses didn't induce any important changes within the biochemical parameters or the histological image. The combined treatment showed important enhancements altogether tested parameters and histological foot age within the liver tissues. Moreover, this improvement was additional pronounced within the cluster received the high dose of the oil [34].

\subsection{Anti-Bacterial Activity}

The essential oils obtained from Thymus vulgaris harvested at 4 biological process stages were evaluated for their biological activity and chemical components. The thyme volatile oils were analyzed for their inhibition effects against 9 strains of gram-negative bacteria and 6 strains of gram-positive bacteria. The bio-impedance methodology was chosen for finding out the antibacterial activity of the essential oils and also the parameter chosen for outlining and quantifying the antibacterial activity of the thyme oils was the detection time. The plate counting technique was used to studying the inhibitory effect by direct exposure. All the thyme essential oils examined had significant bacteria static activity against the microorganisms tested. This activity was additional marked against the gram-positive bacteria. The oil from thyme fully flower was the most effective at stopping the growth of the microorganism species examined. The oils tested were conjointly shown to possess smart antibacterial activity by direct contact, that gave the impression to be a lot of marked against the gram-negative microorganism. Some a number of the species were capable of recovering a minimum of $50 \%$ of their metabolic function once contact with the inhibitor, whereas most of the strains were shown to have been inactivated almost completely [35].

\subsection{Anti-Viral Property}

Silke Nolkemper et al. conducted an experiment with aqueous extracts from species of the Lamiaceae family were examined for their antiviral activity against Herpes simplex virus (HSV). Extracts from thyme (Thymus vulgaris) has shown inhibitory activity against Herpes simplex virus type 1 (HSV-1), type 2 (HSV-2) and an acyclovir-resistant strain of HSV-1 was tested in vitro on RC-37 cells in a plaque reduction assay [36].

\subsection{Anti-Inflammatory Property}

Thymus vulgaris oil is a combination of monoterpenes. The most compounds of this oil are the natural terpenoid thymol and its phenol chemical compound carvacrol [34] that has medicinal drug, anti-oxidative, antimicrobial, anti-tissue, antispasmodic, and antibacterial effects [16]. Terpenoids, flavonoide glycosides, and synthetic resin acids were additionally found in Thymus spp. 


\subsection{Insecticidal Activity}

The insecticidal activity of thyme volatile oil, thymol and carvacrol was evaluated in laboratory against completely different larval stages of lesser meal worm. The sooner and later larval stages were reared on diets containing one or two acetone solutions of tested compounds. Insecticidal activity of thyme volatile oil and pure monoterpenes against larvae relied on the dose and age of larvae. The growth of younger larvae was considerably affected, whereas those of older larval stage were less influenced and only by pure oil components. In young larvae the application $1 \%$ thyme oil, thymol and carvacrol, caused mortality of $50.0 \%$, $86.67 \%$ and $85 \%$, respectively [37].

\section{Conclusion}

While centuries of use in traditional settings can be used as testimony that a particular herbal ingredient is effective or safe, several problems must be addressed as these ingredients are incorporated into modern practice. One problem is that ingredients once used for symptomatic management in traditional healing are now used in developed countries as part of health promotion or disease prevention strategies; thus, acute treatment has been replaced by chronic exposure. This means that a statement about "thousands of years of evidence that a product is safe" may not be valid for the way the product is now being used. This does not expressly mean that an ingredient is unsafe; it does mean that safety in the modern context cannot be assumed. A second problem is that efficacy and effectiveness have rarely been demonstrated using modern scientific investigations. An evidencebased approach to this issue has only recently been implemented, and the results reveal that for most herbal products, considerable gaps in knowledge need to be remedied before one can be convinced about their efficacy. Is traditional medicine reality essential in the world? The more salient problems associated with "modern" medicine are as follows: 1) Its indiscriminative and aggressive dispossession of local health and medical knowledge accumulated by trial and error over many centuries and in some cases millennia; 2) its systematic displacement of the potential for genuine local control and familial participation in the key processes of prevention and cure; 3) it's admitted in capacity to reverse some infectious, and all degenerative and autoimmune diseases; and 4) its swelling wake of (largely unmonitored and uncompensated) problems due to its chosen methods of prevention and cure. The outset of the new millennium the World Health Organization (WHO) estimated that the majority of the populations of most countries were still relying primarily upon indigenous or traditional forms of medicine for meeting every day health care needs. It has been estimated that in developed countries such as United States, plant drugs constitute as much as $25 \%$ of the total drugs, while in fast developing countries such as China and India, the contribution is as much as $80 \%$. Consumers have reported positive attitudes towards these products, in large part because they believe them to be of "natural" rather than "synthetic" origin, they believe that such products are likely to be more safe than drugs, they are considered part of a healthy life style, and they can help to avoid unnecessary contact with conventional "western" medicine. Although modern medicine may exist side-by-side with such traditional practice, herbal medicines have often maintained their popularity for historical and cultural reasons.

\section{References}

[1] Prakash, P. and Gupta, N. (2005) Therapeutic Uses of Ocimum sanctum Linn (Tulsi) with a Note on Eugenol and Its Pharmacological Actions: A Review. Indian Journal of Physiology and Pharmacology, 49, 125-131.

[2] Farnsworth, N.R. (1990) The Role of Ethno Pharmacology in Drug Development. Ciba Foundation Symposium 154. Bioactive Compounds from Plants. John Wiley \& Sons, Baffins Lane, Chichester (England), 2-21.

[3] Vishwakarma, A.P., Vishwe, A., Sahu, P. and Chaurasiya, A. (2013) Magical Remedies of Terminalia arjuna (ROXB.). International Journal of Pharmaceutical Archive, 2, 189-201.

[4] Newman, D.J., Crag, G.M. and Snader, K.M. (2000) The Influence of Natural Products upon Drug Discovery. Natural Product Reports, 17, 215-234. http://dx.doi.org/10.1039/a902202c

[5] Jones, W.P., Chin, Y.-W. and Kinghorn, A.D. (2006) The Role of Pharmachology in Modern Medicine and Pharmacy. Current Drug Targets, 7, 247-264. http://dx.doi.org/10.2174/138945006776054915

[6] Newman, D.J. and Crag, G.M. (2007) Natural Products as Sources of New Drugs over the Last 25 Years. Journal of Natural Products, 70, 461-477. http://dx.doi.org/10.1021/np068054v

[7] Clark, A.M. (1996) Natural Products as a Source for New Drugs. Pharmaceutical Research, 13, 1133-1141. http://dx.doi.org/10.1023/A:1016091631721 
[8] WHO (1993) Research Guidelines for Evaluating the Safety and Efficacy of Herbal Medicines. Manila.

[9] Christopher, B. (2008) RHS A-Z Encyclopedia of Garden Plants. Dorling Kindersley, United Kingdom.

[10] http://bioweb.uwlax.edu/bio203/s2012/disrud_sama/classification.htm

[11] Xiao, P. (1988) A Pictorial Encyclopaedia of Chinese Medicine. v1-10. Commercial Press, Hong Kong, 88-90.

[12] Li, L. (2000) Opportunity and Challenge of Traditional Chinese Medicine in Face of the Entrance to WTO (World Trade Organization). Chinese Journal of Information on Traditional Chinese Medicine, 7, 7-8.

[13] Saito, H. (2000) Regulation of Herbal Medicines in Japan. Pharmacological Research, 41, 515-519. http://dx.doi.org/10.1006/phrs.1999.0645

[14] Arash, R., Koshy, P. and Sekaran, M. (2010) Antioxidant Potential and Phenol Content of Ethanol Extract of Selected Malaysian Plants. Research Journal of Biotechnology, 5, 16-19.

[15] Tippo, O. and Stern, W.L. (1977) Humanistic Botany. W.W. Norton, New York.

[16] Taylor, L. (2000) Plant Based Drugs and Medicines. Raintree Nutrition Inc., Carson City, NV.

[17] Tyler, V.E. (1999) Phytomedicines: Back to the Future. Journal of Natural Products, 62, 1589-1592. http://dx.doi.org/10.1021/np9904049

[18] ESCOP (European Scientific Cooperative on Phytotherapy) (1999) ESCOP Monographs on the Medicinal Uses of Plant Drugs, Exeter, UK.

[19] Blumenthal, M., Busse, W.R., Goldberg, A., Gruenwald, J., Hall, T., Riggins, C.W. and Rister, R.S. (1998) The Complete German Commission E Monographs: Therapeutic Guide to Herbal Medicines, Austin, TX/Boston, MA, American Botanical Council/Integrative Medicine Communications.

[20] WHO (1999) WHO Monographs on Selected Medicinal Plants, Vol. 1, Geneva.

[21] Fumonisin, B. (2002) Some Traditional Herbal Medicines, Some Mycotoxins, Naphthalene and Styrene. IARC Monographs on the Evaluation of Carcinogenic Risks to Humans 82.

[22] Schulz, V., Hänsel, R. and Tyler, V.E. (2001) Rational Phytotherapy. A Physician’s Guide to Herbal Medicine. 4th Edition, Springer-Verlag, Berlin. http://dx.doi.org/10.1007/978-3-642-98093-0

[23] Donald, P.B. (2000) Medicinal Plants and Phytomedicines. Linking Plant Biochemistry and Physiology to Human Health. Plant Physiology, 124, 507-514. http://dx.doi.org/10.1104/pp.124.2.507

[24] Cronquist, A. (1988) The Evolution and Classification of Flowering Plants. New York Botanical Garden, Bronx.

[25] Namsa, N.D., Tag, H., Mandal, M., Kalita, P. and Das, A.K. (2009) An Ethnobotanical Study of Traditional Anti-Inflammatory Plants Used by the Lohit Community of Arunachal Pradesh, India. Journal of Ethnopharmacology, 125, 234-245. http://dx.doi.org/10.1016/j.jep.2009.07.004

[26] Verpoorte, R. (2000) Pharmacognosy in the New Millennium: Leadfinding and Biotechnology. Journal of Pharmacy and Pharmacology, 52, 253-262. http://dx.doi.org/10.1211/0022357001773931

[27] Thompson, J., Chalcha, J., Michet, A., Linhart, Y. and Ehlers, B. (2003) Qualitative and Quantitative Variation in Monoterpene Co-Occurrence and Composition in the Essential Oil of Thymus vulgaris Chemotypes. Journal of Chemical Ecology, 29, 859-880. http://dx.doi.org/10.1023/A:1022927615442

[28] Zarzuelo, A. and Crespo, E. (2002) The Medicinal and Non Medicinal Uses of Thyme. Thyme: The Genus Thymus, 263-292.

[29] Marculescu, A., Vlase, L., Hanganu, D., Dragulescu, C., Antonie, I. and Neli-Kinga, O. (2007) Polyphenols Analyses from Thymus Species. Proceedings of the Romanian Academy-Series B, 3, 117-121.

[30] Maher Ali Ahmed, M., Saeed Mohammed, A. and Ebtesam Hasan, A. (2011) Chemical Composition and Antimicrobial Activity of Essential Oil of Thymus vulgaris from Yemen. Turkish Journal of Biochemistry, 36, 342-349.

[31] Amiri, H. (2012) Essential Oils Composition and Antioxidant Properties of Three Thymus Species. Evidence-Based Complementary and Alternative Medicine, 2012, Article ID: 728065. http://dx.doi.org/10.1155/2012/728065

[32] ESCOP (2007) ESCOP Monographs: The Scientific Foundation for Herbal Medicinal Products. The European Scientific Cooperative on Phytotherapy in Collaboration with Georg Thieme.

[33] El-Nekeety, A.A., Mohamed, S.R., Hathout, A.S., Hassan, N.S., Aly, S.E. and Abdel-Wahhab, M.A. (2011) Antioxidant Properties of Thymus vulgaris Oil against Aflatoxin-Induce Oxidative Stress in Male Rats. Toxicon, 57, 984-991. http://dx.doi.org/10.1016/j.toxicon.2011.03.021

[34] Nickavar, B., Mojab, F. and Dolat-Abadi, R. (2005) Analysis of the Essential Oils of two Thymus Species from Iran. Food Chemistry, 90, 609-611. http://dx.doi.org/10.1016/j.foodchem.2004.04.020

[35] Marino, M. and Bersani, C. (1999) Antimicrobial Activity of the Essential Oils of Thymus vulgaris L. Measured Using a Bioimpedometric Method. Journal of Food Protection, 62, 1017-1023. 
[36] Nolkemper, S., Reichling, J., Stintzing, F.C., Carle, R. and Schnitzler, P. (2006) Antiviral Effect of Aqueous Extracts from Species of the Lamiaceae Family against Herpes simplex Virus Type 1 and Type 2 in Vitro. Planta Medica, 72, 1378-1382. http://dx.doi.org/10.1055/s-2006-951719

[37] Szczepanik, M., Zawitowska, B. and Szumny, A. (2012) Insecticidal Activities of Thymus vulgaris Essential Oil and Its Components (Thymol and Carvacrol) against Larvae of Lesser Mealworm, Alphitobiusdiaperinus Panzer (Coleoptera: Tenebrionidae). Allelopathy Journal, 30, 129-142. 